International Journal of Information Technology, Modeling and Computing (IJTTMC) Vol. 4, No.4, November 2016

\title{
On Estimation of Time Scales of MAss Trans- PORT IN INHOMOGENOUS MATERIAL
}

\author{
E.L. Pankratov and E.A. Bulaeva \\ Nizhny Novgorod State University, 23 Gagarin avenue, Nizhny Novgorod, 603950, \\ Russia
}

\begin{abstract}
In this paper we generalized recently introduced approach of estimation of time scales of mass transport in inhomogenous materials under influence of inhomogenous potential field. Some examples of using of the approach were considered.
\end{abstract}

\section{INTRODUCTION}

Elaboration of solid state electronic devices leads to development of new and optimization of existing technological processes. Due to the elaboration one can obtain increasing of integration rate of elements of integrated circuits, their reliability and frequency characteristics. To develop new and optimization of existing technological processes it is attracted of interest estimation of time characteristics of mass transport: continuance of technological processes and their single steps. It is also attracted an interest estimation of time characteristics during functioning of electronic devices (for example, switching times of diodes et al). In this paper as a development of works [1-6] we introduce an approach to estimate time characteristics of mass transport in multidimensional materials for different boundary conditions. The consider situation gives a possibility to take into account both technological processes of solid state electronics devices and charge carriers transport during functioning of the above devices. We consider most simple to illustrate the approach situation of radial symmetry of mass transport in $N+1$-dimensional material $(N=0,1,2)$. Diffusion coefficient $D(r)$ of charge carriers, dopants, et al is an arbitrary function of radius $r, 0 \leq r \leq a$. At the same time an arbitrary distribution of potential profile as a function of radius $r, 0 \leq r \leq a$, is also presented in the considered material. Framework the paper we consider:

(i) a matter (for example, dopant) with known initial distribution $C(r, 0)=f(r)$ and unit mass $M=1$ existing in the considered material with insulated external boundary $r=a$;

(ii) a matter (for example, excess charge carriers escaping the considered material) with known initial distribution $C(r, 0)=f(r)$ and unit mass $M=1$ existing in the considered material with drain on external boundary $r=a$;

(iii) a source of a matter with constant concentration $C(0, t)=C_{0}$ have been manufactured in central part of material with drain on external boundary $r=a$. In this case with time one can find stationary flow of the considered matter (for example, current density of charge carriers).

At initial moment of time $(t=0)$ one can find starting of relaxation of distribution of concentration of the considered matter. Framework this paper we determine relaxation time of matter as a function of radius $r$ from center in the considered material. As relaxation time in the case (ii) it is practicably to consider escape time of mass of matter. In the cases (i) and (iii) we consider the required time scale as relaxation time of concentration of the considered matter. 
International Journal of Information Technology, Modeling and Computing (IJITMC) Vol. 4, No.4, November 2016

\section{Method Of Solution}

We determine variation of concentration $C(r, t)$ in space and time as solution of the second Fick's law

$$
\frac{\partial C(r, t)}{\partial t}=\operatorname{div}[D(r)\{C(r, t) \operatorname{grad} \varphi(r)+\operatorname{grad} C(r, t)\}]=-\operatorname{div} \vec{G}(r, t)
$$

Here function $\vec{G}(r, t)$ is flow of matter. The flow is proportional to gradient of concentration

$$
\vec{G}(r, t)=-D(r)[C(r, t) \operatorname{grad} \varphi(r)+\operatorname{grad} C(r, t)] .
$$

Boundary and initial conditions for Eq.(1) could be written as

(i) $\vec{G}(0, t)=0, C(r, 0)=f(r), \vec{G}(a, t)=0$;

(ii) $\vec{G}(0, t)=0, C(a, t)=0, C(r, 0)=f(r)$;

(iii) $C(0,0)=C_{0}, C(a, t)=0, C(r, 0)=0$.

We determine relaxation time of distribution of considered matter framework asymptotically optimal $[5,6]$ criterion as equal-sized by square rectangle [1-6]

(i) and (iii)

$$
\Theta_{C}(r)=\frac{\int_{0}^{\infty}[C(r, \infty)-C(r, t)] d t}{C(r, \infty)-C(r, 0)},
$$

$$
\Theta_{M}=M^{-1}(0) \int_{0}^{\infty} M(t) d t
$$

Here $\Theta_{C}(r)$ is the relaxation time of concentration of matter, $\Theta_{M}$ is the escape time of mass of matter. Mass of matter could be estimated by integration of appropriate concentration

$$
M(t)=\int_{V} C(r, t) d V
$$

Here $V$ is the volume of localization of matter. Accounting of radial symmetry transforms the above relation to the following form

$$
M(t)=\gamma \int_{0}^{a} r^{N} C(r, t) d r
$$

Here $\gamma$ is angle normalization. $\gamma$ is equal to unity in the one dimensional case; $\gamma$ is equal to $2 \pi$ in the two dimensional case; $\gamma$ is equal to $4 \pi$ in the two dimensional case.

It should be noted, that criterion for estimation of relaxation time as equal-sized by square rectangle could be used for analysis of monotonous in time concentration of matter [6]. If concentration of matter is nonmonotonous in time, it should be used the recently introduced nonlinear criterion $[5,6]$. 
International Journal of Information Technology, Modeling and Computing (IJTMC) Vol. 4, No.4, November 2016

To calculate the relaxation times by using the criteria Eqs. (3) and (4) we used the Laplace transformations on time of concentration, mass and flow of matter [7]

$$
\hat{M}(s)=\int_{0}^{\infty} M(t) e^{-s t} d t, Y(r, s)=\int_{0}^{\infty} C(r, t) e^{-s t} d t, \hat{G}(r, s)=\int_{0}^{\infty} G(r, t) e^{-s t} d t .
$$

The Laplace transformations of the Eq. (1) and relations (3) and (4) with account initial conditions could be written as

$$
\begin{aligned}
& \operatorname{div} D(r)[\operatorname{grad} \varphi(r) Y(r, s)+\operatorname{grad} Y(r, s)]-s Y(r, s)=-f(r), \\
& \Theta_{C}(r)=\lim _{s \rightarrow 0} \frac{C(r, \infty)-s Y(r, s)}{s[C(r, \infty)-C(r, 0)]}, \\
& \Theta_{M}=\lim _{s \rightarrow 0} M(s) .
\end{aligned}
$$

It should be noted, that it is not necessary to calculate concentration, mass and flow of matter during determination the relaxation times (7) and (8) for all values of the Laplace parameter $s$. They are attracted an interest only asymptotic behaviors of the above values for $s \rightarrow 0$. Let us to decompose functions $s Y(r, s)$ and $s G(r, s)$ in area of small values of parameter $s$ into the following power series

$$
s Y(r, s)=Z_{0}(r)+s Z_{1}(r)+s^{2} Z_{2}(r)+\ldots, s G(r, s)=H_{0}(r)+s H_{1}(r)+s^{2} H_{2}(r)+\ldots
$$

Substitution of the series (9) into relations (7) and (8) leads to the following result

$$
\begin{aligned}
& \Theta_{C}(r)=\lim _{s \rightarrow 0} \frac{C(r, \infty)-Z_{0}(r)-s Z_{1}(r)-s^{2} Z_{2}(r)-\ldots}{s[C(r, \infty)-C(r, 0)]}, \\
& \Theta=\lim _{s \rightarrow 0} \frac{\gamma}{s} \int_{0}^{a}\left(Z_{0}(r)+s Z_{1}(r)+s^{2} Z_{2}(r)+\ldots\right) d r .
\end{aligned}
$$

Limiting theorems of Laplace transformation [7] gives a possibility to obtain the following relations

$$
\lim _{s \rightarrow 0} s Y(r, s)=C(r, \infty)=Z_{0}(r), \lim _{s \rightarrow 0} s G(r, s)=G(r, \infty)=H_{0}(r)
$$

In this situation stationary values of flow and concentrations of matter are equal to $H_{0}(r)=0$ and $Z_{0}(r)$. Calculation of limits of (10) and (11) with account relations (12) gives a possibility to transform relaxation times (7) and (8) to the following form

$$
\begin{aligned}
& \Theta_{C}(r)=\frac{Z_{1}(r)}{f(r)-Z_{0}(r)}, \\
& \Theta_{M}=\gamma \int_{0}^{a} r^{N} Z_{1}(r) d r .
\end{aligned}
$$

To determine relaxation times one shall calculate functions $Z_{0}(r)$ and $Z_{1}(r)$. Substitution of the relation (9) into the equation (6) gives the following system of equations for the functions $Z_{k}(r)$ 
International Journal of Information Technology, Modeling and Computing (IJITMC) Vol. 4, No.4, November 2016 and $H_{k}(r)$

$$
\left\{\begin{array}{l}
\frac{1}{r^{N}} \frac{d}{d r}\left[r^{N} D(r)\left\{\frac{d Z_{0}(r)}{d r}+\frac{d \varphi(r)}{d r} \cdot Z_{0}(r)\right\}\right]=0 \\
\frac{1}{r^{N}} \frac{d}{d r}\left[r^{N} D(r)\left\{\frac{d Z_{1}(r)}{d r}+\frac{d \varphi(r)}{d r} \cdot Z_{1}(r)\right\}\right]=Z_{0}(r)-f(r) \\
\frac{1}{r^{N}} \frac{d}{d r}\left[r^{N} D(r)\left\{\frac{d Z_{k}(r)}{d r}+\frac{d \varphi(r)}{d r} \cdot Z_{k}(r)\right\}\right]=Z_{k-1}(r), k \geq 2
\end{array}\right.
$$

Functions $Z_{k}(r)$ and $H_{k}(r)$ are correlated by the following relation

$$
\frac{d Z_{k}(r)}{d r}+\frac{d \varphi(r)}{d r} Z_{k}(r)=-\frac{H_{k}(r)}{D(r)} .
$$

The first equation of the system (15) could be solved by using standard approaches. Function $Z_{0}(r)$ for different boundary and initial conditions could be written as for the case $(i)$

$$
Z_{0}(r)=e^{-\varphi(r)} /[\gamma \mu(a)]
$$

where $\mu(a)=\int_{0}^{a} r^{N} e^{-\varphi(r)} d r$

for the case (ii)

$$
Z_{0}(r)=0 \text {, }
$$

for the case (iii)

$$
Z_{0}(r)=C_{0} e^{\varphi(0)-\varphi(r)}[\mu(a)-\mu(r)] / \mu(a) .
$$

The second equation of the system (15) could be solved by using standard appro-aches. Function $Z_{0}(r)$ for different boundary and initial conditions could be written as

for the case (i) $Z_{1}(r)=e^{-\varphi(r)}\left[\int_{r}^{a} Q(v) F(v) d v-\int_{0}^{a} Q(v) I(v) F(v) d v-\right.$

$$
\left.-\frac{1}{\gamma} \int_{r}^{a} Q(v) I(v) d v+\frac{1}{\gamma} \int_{0}^{a} I^{2}(v) Q(v) d v\right],
$$

where, $Q(r)=e^{\varphi(r)} / r^{N} D(r), F(x)=\int_{0}^{x} v^{N} f(v) d v, S(r)=\int_{0}^{r} Q(v) d v / \int_{0}^{a} Q(y) d y, I(r)=\mu(r) / \mu(a)$,

for the case (ii) $\quad Z_{1}(r)=e^{-\varphi(r)} \int_{r}^{a} \frac{e^{\varphi(v)}}{v^{N} D(v)} \int_{0}^{v} u^{N} f(u) d u d v$,

for the case (iii) $Z_{1}(r)=C_{0} e^{\varphi(0)-\varphi(r)}\left[\int_{0}^{r} Q(y) \int_{0}^{y} v^{N} e^{-\varphi(v)} \int_{v}^{a} Q(x) d x d v d y-\right.$

$$
\left.-S(r) \int_{0}^{a} Q(y) \int_{0}^{y} v^{N} e^{-\varphi(v)} \int_{v}^{a} Q(x) d x d v d y\right] / \int_{0}^{a} Q(y) d y
$$

Substitution of functions $Z_{0}(r)$ and $Z_{1}(r)$ into relations (13) and (14) gives a possibility to obtain 
International Journal of Information Technology, Modeling and Computing (IJITMC) Vol. 4, No.4, November 2016 relations for relaxation times in the following form

$$
\begin{array}{r}
\text { for the case }(i) \Theta_{C}(r)=\frac{1}{\gamma f(r) e^{\varphi(r)} \mu(a)-1}\left[\gamma \mu(a) \int_{r}^{a} Q(v) F(v) d v-\right. \\
\left.-\gamma \int_{0}^{a} \mu(v) Q(v) F(v) d v-\int_{r}^{a} Q(v) \mu(v) d v+\int_{0}^{a} I(v) \mu(v) Q(v) d v\right] \\
\Theta_{M}=\gamma \int_{0}^{a} \frac{e^{\varphi(v)} \mu(v)}{v^{N} D(v)} F(v) d v .
\end{array}
$$

for the case (ii) $\Theta_{M}=\gamma \int_{0}^{a} \frac{e^{\varphi(v)} \mu(v)}{v^{N} D(v)} F(v) d v$,

for the case (iii) $\Theta_{C}(r)=\left[S(r) \int_{0}^{a} Q(y) \int_{0}^{y} v^{N} e^{-\varphi(v)} \int_{v}^{a} Q(x) d x d v d y-\right.$

$$
\left.-\int_{0}^{r} Q(y) \int_{0}^{v} v^{N} e^{-\varphi(v)} \int_{v}^{a} Q(x) d x d v d y\right] / \int_{r}^{a} Q(v) d v
$$

\section{Examples Of Calculation Of Relaxation Time}

\section{Example of initial distribution}

For materials without source we consider the following initial distribution of matter: $f(r)=\delta(r)$. In this case relaxation times (20) and (21) could be written as

for the case $(i) \Theta_{C}(r)=\left[\gamma \int_{0}^{a} \mu(v) Q(v) d v-\gamma \mu(a) \int_{r}^{a} Q(v) d v+\right.$

$$
\left.+\int_{r}^{a} Q(v) \mu(v) d v-\int_{0}^{a} I(v) \mu(v) Q(v) d v\right]
$$

for the case $(i i)$

$$
\Theta_{M \delta}=\gamma \int_{0}^{a} \mu(v) Q(v) d v .
$$

In future we will use only considered initial distribution of matter.

\section{$\underline{\text { Example of potential profile } 1}$}

We consider free diffusion, when potential profile is constant for any point of material $\varphi(r)=\varphi_{0}$. In this case relaxation times (22)-(24) will not dependent of potential. In this case these relaxation times (22)-(24) could be written as

for the case $(i) \Theta_{C \delta}(r)=\frac{\gamma}{N+1} \int_{0}^{a} \frac{v d v}{D(v)}-\frac{\gamma a^{N+1}}{N+1} \int_{r}^{a} \frac{d v}{v^{N} D(v)}+$

$$
+\frac{1}{N+1} \int_{r}^{a} \frac{v d v}{D(v)}-\frac{1}{(N+1) a^{N+1}} \int_{0}^{a} \frac{v^{N+2} d v}{D(v)}
$$

for the case (ii)

$$
\Theta_{M \delta}=\frac{\gamma}{N+1} \int_{0}^{a} \frac{v d v}{D(v)},
$$


International Journal of Information Technology, Modeling and Computing (IJITMC) Vol. 4, No.4, November 2016

for the case (iii)

$$
\begin{aligned}
& \text { case (iii) } \Theta_{C}(r)=\left\{\left[\int_{0}^{a} \frac{d v}{v^{N} D(v)}\right]_{0}^{-1} \int_{0}^{r} \frac{d v}{v^{N} D(v)} \int_{0}^{a} \frac{1}{y^{N} D(y)} \times\right. \\
& \left.\times \int_{0}^{y} v^{N} e^{-\varphi(v)} \int_{v}^{a} \frac{d x d v d y}{x^{N} D(x)}-\int_{0}^{r} \frac{1}{y^{N} D(y)} \int_{0}^{y} v^{N} e^{-\varphi(v)} \int_{v}^{a} \frac{d x d v d y}{x^{N} D(x)}\right\} / \int_{r}^{a} \frac{d v}{v^{N} D(v)} .
\end{aligned}
$$

In the simplest case of constant diffusion coefficient $D(r)=D_{0}$ relations (25)-(27) takes the form

for the case $(i) \Theta_{c \delta}(r)=\frac{a^{2} \gamma}{2(N+1) D_{0}}-\frac{\gamma a^{N+1}\left(a^{1-N}-r^{1-N}\right)}{\left(1-N^{2}\right) D_{0}}+$

$$
\begin{array}{r}
+\frac{a^{2}-r^{2}}{2(N+1) D_{0}}-\frac{a^{2}}{(N+1)(N+3) D_{0}}, N \neq 1 \\
\Theta_{C \delta}(r)=\frac{\gamma a^{2}}{4 D_{0}}\left[1-2 \ln \left(\frac{a}{r}\right)\right]+\frac{a^{2}-r^{2}}{4 D_{0}}-\frac{a^{2}}{8 D_{0}}, N=1
\end{array}
$$

for the case (ii)

$$
\Theta_{M \delta}=\frac{\gamma a^{2}}{(N+1) D_{0}}
$$

for the case (iii) $\Theta_{C}(r)=\frac{1}{\left(a^{1-N}-r^{1-N}\right) D_{0}}\left[\frac{r^{1-N} a^{2}(1-N)}{(1+N)(3-N)}-\frac{r^{2} a^{1-N}}{2(1+N)}+\frac{r^{3-N}}{3-N}\right]$

$$
\Theta_{C}(r)=\frac{r^{2}}{4 a^{2} D_{0}} \ln \left(\frac{a}{r}\right), N=1 .
$$

The relation (28) shows that the relaxation time of a matter concentration or mass in a material with insulated external boundaries increases with increasing of value of radius $r$. At small values of radius $r$ concentration of the matter lose monotonous behavior in time. In this case to determine the relaxation time it should be used recently introduced nonlinear criterion. Dependence of the relaxation time (30) is nonmonotonous function of radius with zero values on external boundary of the material $r=a$ at zero value of radius $r$ (i.e. at $r=0$ ).

In the present time one could find wide using different multilayer structures (see, for example, [8]). We consider simplest multilayer structure with diffusion coefficient $D(r)=D_{1}[1(r)-1(r-$ $\left.\left.r_{1}\right)\right]+D_{2} 1\left(r-r_{1}\right)$, where $1(r-\alpha)$ is the unit step-wise function [7]. In the considered materials, which includes into itself the above two layers, the relaxation times (25)-(27) could be written as the case $(i)$

$$
\begin{aligned}
\Theta_{c \delta}\left(r<r_{1}, N \neq 1\right)=\frac{1+\gamma}{2(1+N)}\left(\frac{r_{1}^{2}}{D_{1}}+\frac{a^{2}-r_{1}^{2}}{D_{2}}\right)-\frac{1}{a^{N+1}(1+N)(3+N)} \times \\
\quad \times\left(\frac{r_{1}^{N+3}}{D_{1}}+\frac{a^{N+3}-r_{1}^{N+3}}{D_{2}}\right)-\frac{\gamma a^{N+1}}{1-N^{2}}\left(\frac{r_{1}^{1-N}}{D_{1}}+\frac{a^{1-N}-r_{1}^{1-N}}{D_{2}}\right)-\frac{r^{2}}{2(N+1) D_{1}},
\end{aligned}
$$


International Journal of Information Technology, Modeling and Computing (IJITMC) Vol. 4, No.4, November 2016

$\begin{aligned} \Theta_{C \delta}\left(r>r_{1}, N \neq 1\right)= & \frac{1}{2(N+1)}\left[\frac{\gamma r_{1}^{2}}{D_{1}}+\frac{(1+\gamma) a^{2}-r_{1}^{2}-r^{2}}{D_{2}}\right]- \\ & -\frac{\left(a^{N+3}-r^{N+3}\right)}{a^{N+1}(1+N)(3+N) D_{2}}-\frac{\gamma a^{N+1}}{1-N^{2}} \frac{\left(a^{1-N}-r^{1-N}\right)}{D_{2}},\end{aligned}$

$\Theta_{C \delta}\left(r<r_{1}, N=1\right)=\frac{r_{1}^{2}}{4 D_{1}}+\frac{a^{2}(1+\gamma)-r_{1}^{2}-r^{2}}{4 D_{2}}-\frac{1}{8 a^{2}}\left(\frac{r_{1}^{4}}{D_{1}}+\frac{a^{4}-r_{1}^{4}}{D_{2}}\right)-$

$$
-\frac{\gamma a^{2}}{2}\left[\frac{1}{D_{1}} \ln \left(\frac{r_{1}}{r}\right)+\frac{1}{D_{2}} \ln \left(\frac{a}{r_{1}}\right)\right]
$$

$\Theta_{C \delta}\left(r>r_{1}, N=1\right)=\frac{1}{4}\left[\frac{\gamma r_{1}^{2}}{D_{1}}+\frac{(0,5+\gamma) a^{2}-r_{1}^{2}}{D_{2}}\right]-$

$$
-\frac{1}{8 a^{2}}\left(\frac{r_{1}^{4}}{D_{1}}+\frac{a^{4}-r_{1}^{4}}{D_{2}}\right)-\frac{\gamma a^{2}}{2 D_{2}} \ln \left(\frac{a}{r}\right)-\frac{r^{2}}{4 D_{2}},
$$

for the case (ii)

$$
\Theta_{M \delta}=(N+1) \frac{\gamma}{2}\left(\frac{r_{1}^{2}}{D_{1}}+\frac{a^{2}-r_{1}^{2}}{D_{2}}\right)
$$

for the case (iii) $\Theta_{C \delta}\left(r<r_{1}, N \neq 1\right)=\left\{\frac{D_{2} r^{1-N}}{D_{2} r_{1}^{1-N}+D_{1}\left(a^{1-N}-r_{1}^{1-N}\right)}\left[\frac{1}{2(1+N)}\left(\frac{r_{1}^{3-N}}{D_{1}^{2}}-\right.\right.\right.$

$$
\begin{aligned}
& \left.\left.-\frac{a^{2} r_{1}^{1-N}}{D_{2}^{2}}+\frac{r_{1}^{1-N} a^{2}+r_{1}^{2} a^{1-N}-2 r_{1}^{3-N}}{D_{1} D_{2}}-\frac{(3-N) r_{1}^{3-N}}{D_{2}^{2}}+\frac{r_{1}^{3-N}}{D_{2}^{2}}\right)\right]-\frac{r^{2}\left[r_{1}^{1-N} D_{2}+\left(a^{1-N-} r_{1}^{1-N}\right)\right]}{2(1+N) D_{1} D_{2}}- \\
& \left.-\frac{r^{3-N}}{2(3-N) D_{1}^{2}}\right\} \frac{D_{1} D_{2}}{\left(r_{1}^{1-N}-r^{1-N}\right) D_{2}+\left(a^{1-N}-r_{1}^{1-N}\right) D_{1}},
\end{aligned}
$$

$$
\begin{gathered}
\Theta_{C \delta}\left(r>r_{1}, N \neq 1\right)=\left\{\frac { D _ { 2 } r _ { 1 } ^ { 1 - N } + D _ { 1 } ( r ^ { 1 - N } - r _ { 1 } ^ { 1 - N } ) } { D _ { 2 } r _ { 1 } ^ { 1 - N } + D _ { 1 } ( a ^ { 1 - N } - r _ { 1 } ^ { 1 - N } ) } \left[\frac { 1 } { 2 ( 1 + N ) } \left(\frac{2 r_{1}^{2}\left(a^{1-N}-r^{1-N}\right)}{D_{1} D_{2}}+\frac{r_{1}^{3-N}}{D_{1}^{2}}+\right.\right.\right. \\
\left.\left.+\frac{a^{3-N}-r_{1}^{2} a^{1-N}-r_{1}^{1-N} a^{2}+r_{1}^{3-N}}{D_{2}^{2}}+\frac{r_{1}^{1-N}\left(a^{2}-r_{1}^{2}\right)}{D_{2}^{2}(2-N)}\right)-\frac{r_{1}^{3-N}}{2(3-N) D_{1}^{2}}\right]-\frac{\left(r^{2}-r_{1}^{2}\right) r_{1}^{1-N}}{2 D_{2}^{2}(1+N)}- \\
-\frac{\left[r_{1}^{2} D_{2}+\left(r^{2}-r_{1}^{2}\right) D_{1}\right]\left[r_{1}^{1-N} D_{2}+\left(a^{1-N-} r_{1}^{1-N}\right) D_{1}\right]}{2(1+N) D_{1}^{2} D_{2}^{2}}+\frac{r^{2} r_{1}^{1-N}}{D_{1} D_{2}(1+N)}-\frac{r^{3-N}(1-N)}{2(3-N)(1+N) D_{1}^{2}}- \\
\left.-\frac{r_{1}^{2} r^{1-N}}{(1+N) D_{1} D_{2}}-\frac{\left(r^{3-N}-r_{1}^{3-N}\right)}{2(3-N) D_{2}^{2}}+\frac{r_{1}^{2}\left(r^{1-N}-r_{1}^{1-N}\right)}{2(1+N) D_{2}^{2}}\right\} \frac{D_{2}}{a^{1-N}-r^{1-N}},
\end{gathered}
$$


International Journal of Information Technology, Modeling and Computing (IJITMC) Vol. 4, No.4, November 2016

$$
\begin{aligned}
& \Theta_{C \delta}\left(r<r_{1}, N=1\right)=\left\{\left[\frac{3 r_{1}^{2} \ln r_{1} / a}{4 D_{1}^{2}}-\frac{r_{1}^{2}}{4 D_{1} D_{2}} \ln \left(\frac{r_{1}}{a}\right)-\frac{a^{2}}{4 D_{2}^{2}} \ln \left(\frac{r_{1}}{a}\right)+\frac{a^{2}\left(1+2 \ln r_{1} / a\right)-r_{1}^{2}}{8 D_{2}^{2}}\right] \times\right. \\
& \left.\times \frac{D_{2} \ln r / r_{1}}{D_{1} \ln a / r_{1}+D_{2} \ln r / r_{1}}-\frac{r^{2}\left(1+\ln r_{1} / r\right)}{4 D_{1}^{2}}-\frac{r^{2} \ln a / r_{1}}{4 D_{1} D_{2}}\right\} \frac{D_{1} D_{2}}{D_{2} \ln r_{1} / r+D_{1} \ln a / r_{1}}, \\
& \Theta_{C \delta}\left(r>r_{1}, N=1\right)=\left\{\frac { D _ { 2 } \operatorname { l n } r _ { 1 } D _ { 1 } \operatorname { l n } r / r _ { 1 } + D _ { 2 } \operatorname { l n } r _ { 1 } / a } { D _ { 1 } \operatorname { l n } a / r _ { 1 } + D _ { 2 } \operatorname { l n } r _ { 1 } / r } \left[\frac{r_{1}^{2}}{4 D_{1}^{2}}\left[3 \ln \left(\frac{a}{r_{1}}\right)+1\right]+\frac{a^{2}-2 r_{1}^{2}}{4 D_{2}^{2}} \ln \left(\frac{a}{r_{1}}\right)+\right.\right. \\
& \left.+\frac{r_{1}^{2} \ln a / r_{1}}{2 D_{1} D_{2}}+\frac{r_{1}^{2}}{2 D_{1}^{2}}+\frac{a^{2}-2 r_{1}^{2}}{2 D_{1} D_{2}}-\frac{\left(a^{2}-r_{1}^{2}\right) \ln a / r_{1}}{2 D_{2}^{2}}+\frac{a^{2}-r_{1}^{2}\left(1+\ln a / r_{1}\right)}{4 D_{2}^{2}}\right]+\frac{r^{2}-r_{1}^{2}}{2 D_{1} D_{2}} \ln \left(\frac{r_{1}}{a}\right)- \\
& \left.-\frac{r_{1}^{2} \ln r_{1} / r}{4 D_{1}^{2}}+\frac{r_{1}^{2} \ln a / r_{1}}{4 D_{1} D_{2}}+\frac{r^{2}-r_{1}^{2}}{2 D_{2}^{2}} \ln \frac{a}{r_{1}}-\frac{r^{2}}{8 D_{2}^{2}}+\frac{r^{2}-r_{1}^{2}}{4 D_{2}^{2}} \ln \frac{r_{1}}{r}-\frac{r^{2}-r_{1}^{2}}{8 D_{1}^{2}}-\frac{r_{1}^{2} \ln \left(r / r_{1}\right)}{4 D_{1} D_{2}}\right\} \times \\
& \times D_{2} \ln ^{-1}\left(\frac{a}{r}\right) .
\end{aligned}
$$

In the case, when $D_{1}=D_{2}$, relations (31)-(33) coincide with relations (28)-(30). It could be shown, that increasing of number of layers in the considered multilayer structure leads to averaging of spatial distribution of diffusion coefficient and possibility to approximate the distribution by it's average value $D_{0}[9]$.

\section{Example of potential profile 2}

Now we consider linear dependence of potential profile of radius $\varphi(r)=\alpha r+\varphi_{0}$. In this situation fully analytical integration in relations for relaxation time (20)-(22) could be done only for onedimension case $(N=0)$. In the case of larger dimensions relations for relaxation time depend on the following functions $E(r)=\int_{0}^{r} v^{-1} e^{\alpha v} d v$ and $E^{*}(r)==\int_{0}^{r} v^{-1} e^{-\alpha v} d v$, which are not integrating analytically [10]. Relations for relaxation times for linear potential and constant diffusion coefficient $D(r)=D_{0}$ could be written as

for the case $(i) \Theta_{C \delta}(r, N=0)=\frac{1}{\alpha^{2} D_{0}}\left[\alpha a(2 \gamma+1)-(\gamma+1) e^{\alpha_{r}}-\right.$

$$
\begin{gathered}
\left.-2 \gamma+\gamma e^{\alpha(a-r)}+\alpha(r-a)-\frac{e^{\alpha a}-e^{-\alpha a}-2-\alpha a}{1-e^{-\alpha a}}\right], \\
\Theta_{C \delta}(r, N=1)=\frac{1}{\alpha^{2} D_{0}}\{E(a)-\gamma \ln (\alpha a)-\alpha[a(1+\gamma)-r]-E(r)(1-\gamma)- \\
\left.-\ln \left(\frac{a}{r}\right)+\gamma e^{-\alpha a}(1+\alpha a)[E(a)-E(r)]\right\}-\frac{1}{\alpha^{2} D_{0}}\left[1-e^{-\alpha a}-\alpha a e^{-\alpha a}+2\left(1-e^{-\alpha a}\right)-\right. \\
\left.-2 \alpha a+E^{*}(a)-\ln (\alpha a)+E(a)\right]\left(1-e^{-\alpha a}-\alpha a e^{-\alpha a}\right)^{-1}, \\
\Theta_{C \delta}(r, N=2)=\frac{1}{\alpha^{2} D_{0}}\left\{2 E(a)(1+\gamma)+\gamma \frac{1-e^{\alpha a}}{\alpha a}-\alpha a(2 \gamma+1)-2 \gamma \ln (\alpha a)+\frac{e^{\alpha r}}{\alpha r}+\right.
\end{gathered}
$$


International Journal of Information Technology, Modeling and Computing (IJITMC) Vol. 4, No.4, November 2016

$$
\begin{aligned}
& +2 \frac{a-r}{\alpha r}+\alpha r-\gamma e^{\alpha r}\left(\frac{1-e^{-\alpha a}}{\alpha r}\right)-\gamma\left(1-e^{-\alpha a}\right)[E(a)-E(r)]+\gamma(1+\alpha a) \frac{a}{r} e^{\alpha(r-a)}-\gamma+ \\
& \left.+2 E(r)+2 \alpha^{2} a^{2} \gamma[E(a)-E(r)] \operatorname{ch}(\alpha a)\right\}-\frac{1}{\alpha^{2} D_{0}}\left\{2\left(1-e^{-\alpha a}\right)-\alpha a(1+\alpha a) e^{-\alpha a}+\right. \\
& +4\left(1-e^{-\alpha a}\right)-4 E^{*}(a)-\frac{4 e^{-\alpha a}}{\alpha^{2} D_{0}}+\frac{8}{\alpha a}-\frac{4 e^{\alpha a}}{\alpha a}-4\left[1-e^{-\alpha a}-\alpha a e^{-\alpha a}\right]+4\left(e^{-\alpha a}-1\right)+ \\
& \left.+E(a)+4 \alpha a+8 E^{*}(a)-8 \ln (\alpha a)\right\}\left[2\left(1-e^{-\alpha a}\right)-\alpha a e^{-\alpha a}(\alpha a+2)\right]^{-1}
\end{aligned}
$$

for the case (ii) $\quad \Theta_{M \delta}(N=0)=\gamma \frac{e^{\alpha a}-1-\alpha a}{\alpha^{2} D_{0}}$,

$$
\Theta_{M \delta}(N=1)=\gamma \frac{E(a)-\ln (\alpha a)-\alpha a}{\alpha^{2} D_{0}},
$$

$$
\Theta_{M \delta}(N=2)=\frac{\gamma}{\alpha^{2} D_{0}}\left\{\frac{2}{\alpha}\left[\alpha E(a)-\frac{e^{\alpha a}}{a}\right]+\frac{2}{a \alpha}-\frac{\ln (\alpha a)}{\alpha}-a \alpha\right\},
$$

for the case (iii) $\Theta_{C}(r, N=0)=\frac{1}{\alpha D_{0}\left(e^{\alpha a}-e^{\alpha r}\right)} \times$

$$
\begin{gathered}
\times\left[\frac{\left(e^{\alpha r}-1\right)}{\left(e^{\alpha a}-1\right)}\left(\left\{e^{2 \alpha a}-1\right\}-2 a \alpha e^{\alpha a}\right)-\left(e^{\alpha(a+r)}+e^{\alpha r}-e^{\alpha a}-1\right)-r \alpha\left(e^{\alpha a}+e^{\alpha r}\right)\right], \\
\Theta_{C}(r, N=1)=\frac{E(r)}{E(a)} \int_{0}^{a} \frac{e^{\alpha \nu}}{v D_{0}} \int_{0}^{v} y e^{-\alpha y}[E(a)-E(y)] d y d v- \\
-\int_{0}^{r} \frac{e^{\alpha v}}{v D_{0}} \int_{0}^{v} y e^{-\alpha y}[E(a)-E(y)] d y d v, \\
\left.\left.+\frac{2 \ln a / r}{\alpha^{2}}+\frac{e^{\alpha a}}{a}-\frac{e^{\alpha r}}{r}-\alpha E(a)\right]-\alpha \int_{0}^{a} \frac{e^{\alpha y}}{y^{2}} \int_{0}^{y} v^{2} E(v) d v d y\right\}- \\
-\frac{1}{D_{0}}\left\{\frac{e^{\alpha r}-1}{2 \alpha}+\left[\frac{e^{\alpha a}}{a}-\alpha E(a)\right]\left[\frac{r}{\alpha}-2 \alpha E(r)\right]-\int_{0}^{r} \frac{e^{\alpha y}}{y^{2}} \int_{0}^{y} v^{2} E(v) e^{-\alpha v} d v d y\right\} .
\end{gathered}
$$

Decreasing of the parameter $\alpha$ leads to decreasing difference between relations (34)-(36) and (28)-(30). 


\section{CONCLUSION}

In this paper we generalized recently introduced approach of estimation of time scales of mass transport in inhomogenous materials under influence of inhomogenous potential field. Some examples of using of the approach were considered.

\section{ACKNOWLEDGMENTS}

This work is supported by the agreement of August 27, 2013 № 02.B.49.21.0003 between The Ministry of education and science of the Russia and Lobachevsky State University of Nizhny Novgorod, educational fellowship for scientific research of Government of Russia, educational fellowship for scientific research of Government of Nizhny Novgorod region of Russia and educational fellowship for scientific research of Nizhny Novgorod State University of Architecture and Civil Engineering.

[1] A.N. Malakhov. Radiophysics and Quantum Electronics. Vol. 40 (7). P. 886-896 (1997).

[2] A.N. Malakhov. Radiophysics and Quantum Electronics. Vol. 42 (6). P. 581-589 (1999).

[3] A.N. Malakhov. Chaos. Vol. 7 (3). P. 488-504 (1997).

[4] A.N. Malakhov, E.L. Pankratov. In Proceedings of 4-th conference of radiophysics. Ed. A.V. Yakimov. Nizhny Novgorod. P. 236-237 (2000).

[5] E.L. Pankratov. Collection of scientific papers "In memory of A.N. Malakhov". Ed. A.I. Saichev. Nizhny Novgorod. P. 109-114 (2000).

[6] A.N. Malakhov, E.L. Pankratov. Radiophysics and Quantum Electronics. Vol. 44 (6). P. 367-373 (2001).

[7] G. Korn, T. Korn. Mathematical Handbook for scientists and engineers. Definitions, theorems and formulas for reference and review. Second edition. McGraw-Hill Book Company. New York, 1968.

[8] A.P. Zhang, G.T. Dang, F. Ren, J. Han, A.G. Baca, R.J. Shul, H. Cho, C. Monier, X.A. Cao, C.R. Abernathy, S.J. Pearton. App. Phys. Lett. Vol. 76 (20). P. $2943-2945$ (2000).

[9] A.A. Mal'tsev, E.L. Pankratov. In Proceedings of 4-th conference of radiophysics. Ed. A.V. Yakimov. Nizhny Novgorod. P. 211-212 (2001).

[10] I.S. Gradshtein, I.M. Ryzhik. Tables of integrals, sums, series and products. Moscow, Science, 1971. 